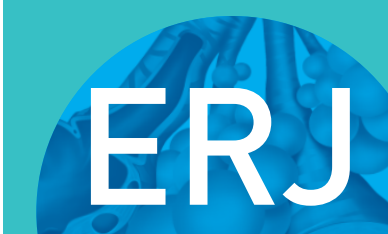

open research

\title{
Ability of the LACE index to predict 30-day hospital readmissions in patients with community-acquired pneumonia
}

\author{
Claudia C. Dobler (1) ${ }^{1,2,3}$, Maryam Hakim ${ }^{2,3}$, Sidhartha Singh ${ }^{2}$, \\ Matthew Jennings ${ }^{4}$, Grant Waterer ${ }^{5}$ and Frances L. Garden ${ }^{3}$
}

Affiliations: ${ }^{1}$ Institute for Evidence-Based Healthcare, Bond University, Gold Coast, Australia. ${ }^{2}$ Dept of Respiratory Medicine, Liverpool Hospital, Sydney, Australia. ${ }^{3}$ South Western Sydney Clinical School, University of New South Wales, Sydney, Australia. ${ }^{4}$ Physiotherapy Dept, Liverpool Hospital, Sydney, Australia. ${ }^{5}$ University of Western Australia, Perth, Australia.

Correspondence: Claudia C. Dobler, Institute for Evidence-Based Healthcare, Bond University, Gold Coast, Queensland 4229, Australia. E-mail: cdoblerlabond.edu.au

\section{ABSTRACT}

Background and objective: Hospital readmissions within 30 days are used as an indicator of quality of hospital care. We aimed to evaluate the ability of the LACE (Length of stay, Acuity of admission, Comorbidities based on Charlson comorbidity score and number of Emergency visits in the last 6 months) index to predict the risk of 30-day readmissions in patients hospitalised for community-acquired pneumonia (CAP).

Methods: In this retrospective cohort study a LACE index score was calculated for patients with a principal diagnosis of CAP admitted to a tertiary hospital in Sydney, Australia. The predictive ability of the LACE score for 30-day readmissions was assessed using receiver operator characteristic curves with C-statistic.

Results: Of 3996 patients admitted to hospital for CAP at least once, 8.0\% ( $n=327)$ died in hospital and $14.6 \%(n=584)$ were readmitted within 30 days. $17.8 \%$ (113 of 636$)$ of all 30-day readmissions were again due to CAP, followed by readmissions for chronic obstructive pulmonary disease, heart failure and chest pain. The LACE index had moderate discriminative ability to predict 30-day readmission (C-statistic $=0.6395$ ) but performed poorly for the prediction of 30 -day readmissions due to CAP (C-statistic=0.5760).

Conclusions: The ability of the LACE index to predict all-cause 30-day hospital readmissions is comparable to more complex pneumonia-specific indices with moderate discrimination. For the prediction of 30-day readmissions due to CAP, the performance of the LACE index and modified risk prediction models using readily available variables (sex, age, specific comorbidities, after-hours, weekend, winter or summer admission) is insufficient.

@ERSpublications

The LACE index is easy to use and its ability to predict all-cause 30-day hospital readmissions for patients hospitalised with community-acquired pneumonia is comparable to more complex pneumonia-specific indices with moderate discrimination https://bit.ly/2SYkxam

Cite this article as: Dobler CC, Hakim M, Singh S, et al. Ability of the LACE index to predict 30-day hospital readmissions in patients with community-acquired pneumonia. ERJ Open Res 2020; 6: 00301-2019 [https://doi.org/10.1183/23120541.00301-2019].

This article has supplementary material available from openres.ersjournals.com

Received: 1 Nov 2019 | Accepted after revision: 5 May 2020

Copyright $\odot$ ERS 2020. This article is open access and distributed under the terms of the Creative Commons Attribution Non-Commercial Licence 4.0. 


\section{Introduction}

Hospital readmissions within 30-days have received a lot of attention in recent years as an indicator of quality of hospital care, and institutions with high readmission rates face financial penalties [1]. The association between 30-day hospital readmissions and quality of care is, however, only poorly supported by evidence. Most readmissions after the seventh day post-hospital discharge may in fact be related to community- and household-level factors beyond hospitals' control [2]. Nevertheless, hospital readmissions are straining healthcare resources [3], interfere with patients' daily living and are a burden to patients $[4,5]$.

The causes for 30-day hospital readmissions are multifaceted, but there seems to be an association with increasing multi-morbidity and thus medical complexity [6] as well as changes in provision of health services with shorter length of stay in hospital and increasing efforts to shift provision of care to an outpatient setting [7, 8]. High 30-day readmissions rates have been reported in chronic obstructive pulmonary disease (COPD) and heart failure, both chronic conditions characterised by episodes of acute worsening [9]. Maybe surprisingly, high 30-day readmission rates have also been described in community-acquired pneumonia (CAP) [10]. In the current study, we assessed risk factors for 30-day hospital readmissions in patients hospitalised with CAP.

Several generic and pneumonia-specific models to predict patients' risk of 30-day hospital readmission have been developed [11, 12]. The goal of these risk prediction models is to identify patients at an increased risk of readmission in order to use targeted interventions such as comprehensive discharge planning to reduce hospital readmissions.

We aimed to evaluate the ability of the LACE index score [13] (Length of stay, Acuity of admission, Comorbidities calculated by Charlson comorbidity score [14] and number of Emergency visits in the last 6 months), a commonly used and easy to use generic risk prediction tool, to predict 30-day all-cause hospital readmissions in a cohort of patients with CAP.

\section{Methods}

We conducted a retrospective cohort study, for which we extracted data from the electronic hospital management system of Liverpool Hospital, a tertiary hospital in Sydney, Australia. The hospital is located in a low socioeconomic area with a high proportion of migrants from culturally and linguistically diverse backgrounds.

The study cohort included all patients aged 18 years and above who were admitted to Liverpool Hospital between January 1, 2006 and January 31, 2016 with a principal diagnosis indicative of CAP at discharge, identified through International Classification of Diseases-10 codes (A40.3, B01.2, J10.0, J11.0, J12.0-3, J12.8-9, J13, J14, J15.0, J15.2, J15.4-9, J16.8, J18.0-1, J18.8-9, J85.1).

We excluded patients with aspiration pneumonia, patients with haematological malignancies and patients who were discharged to a nursing home. We did not aim to differentiate potential cases of healthcare-associated pneumonia (HCAP) from CAP, because evidence from the Asia Pacific region suggests that the choice of antibiotic therapy should be based on patients' individual risk factors rather than applying a HCAP classification system [15].

We randomly chose 30 files of patients who had a hospitalisation for CAP to evaluate the possible risk of diagnosis misclassification (in particular hospital-acquired pneumonia or pneumonia in immunocompromised patients classified as CAP).

Data analysis

A LACE index score was calculated for each patient. All patients with CAP were admitted via the emergency department and had therefore the maximum score of three points for acuity. We used receiver operating characteristic (ROC) curves to assess the predictive ability (sensitivity and specificity) of the LACE index for 1) all-cause readmissions at 30 days; and 2) readmissions due to CAP at 30 days. The discriminative ability of a risk prediction model was expressed as C-statistic, which can take values ranging from 0.5 (no discrimination, no better than chance) to 1.0 (perfect discrimination).

We assessed reasons for readmission after an index hospitalisation for CAP within 30 days as well as 1 year. A readmission was defined as a re-hospitalisation following an index hospitalisation with a principal diagnosis of CAP. Index hospitalisations and readmissions were only captured if they occurred at the study hospital. Readmissions were analysed based on hospitalisation episodes, meaning that a patient could have had more than one index hospitalisation during the study period.

The association between each of the LACE variables and readmission was assessed using a generalised estimating equation. We tested whether the predictive ability of the LACE model could be improved by assessing modifications of the LACE prediction model. This included substituting the three points for 
acuity of admission for: admission during a weekend; admission after core hours; admission during winter or summer season (winter: 1 June to 31 August, summer: 1 December to 28 February in the Southern Hemisphere); the patient being male; specific comorbidities (COPD, heart failure, diabetes, and chronic renal failure); and age $>60,>65,>70,>75$, and $>80$ years respectively. We also tested new prediction models with different combinations of these variables.

A Chi-squared of trend was used to assess if readmission rates increased over time. A generalised estimating equation was used to test whether there was a significant interaction term between readmission rates and length of stay over time. Statistical analysis was conducted using SAS 9.4 (SAS Institute Inc., Cary, NC, USA).

Ethical approval was obtained from the South Western Sydney Local Health District Human Research Ethics Committee (HREC/15/LPOOL/557).

\section{Results}

During the study period 3996 patients had at least one hospitalisation for CAP. These patients accounted for a total of 21171 hospitalisations for various reasons, of which 4508 were for CAP. More than half the patients were male (52\%). At the first admission, the mean age of patients was 66.4 years $(\mathrm{SD}=18.8$; range 18 to 102) and the median Charlson comorbidity index score was 1 (interquartile range (IQR): 0-2, range: 0-44). The most common ICD-10 code for CAP index admissions was J 18.9 (unspecified pneumonia), which made up $85.6 \%(n=3861)$ of all index admissions. Supplementary Table S1 gives an overview of the distribution of ICD-10 codes for CAP index admissions.

\section{Deaths and readmissions}

Of 3996 patients admitted for CAP, 8.0\% $(n=327)$ died in hospital and $14.6 \%(n=584)$ were readmitted for any cause and $2.8 \%(\mathrm{n}=110)$ for CAP within 30 days. After an index hospitalisation for CAP ( $\mathrm{n}=4508)$, the 30 -day readmission rate was $14.1 \%$ (636 out of 4508 hospitalisation episodes). Of the 30 -day readmissions, $113(17.8 \%)$ were again due to CAP, followed by readmissions for COPD (44, 6.9\%), heart failure (42, $6.6 \%)$ and aspiration pneumonia $(22,3.5 \%)$ (table 1). The median time to readmission for CAP for those readmitted within 30 days was 7 days (IQR: 3 to 16). The main reason for 1-year readmissions (1851, 41.1\%) after an index hospitalisation of pneumonia was another episode of pneumonia $(228,12.3 \%)$, followed by COPD $(121,6.5 \%)$, heart failure $(103,5.6 \%)$ and chest pain $(66,3.6 \%)$ (table 1$)$.

The 30-day readmissions for any diagnosis following an index hospitalisation for CAP increased from $11.6 \%$ in 2006 to $15.1 \%$ in 2015 ( $\mathrm{p}$ for trend=0.18) (figure 1). The median length of stay of index hospitalisations significantly decreased from a mean of 5.5 days in 2006 to 4.0 days in 2015 ( $p$ for trend $<0.001$ ) (figure 2) but was not significantly associated with readmissions per year $(\mathrm{p}=0.51)$.

\section{Predicting all-cause readmissions}

The mean LACE score for index admissions of CAP was 8.8 (range 3-19) with a median score of 8 (table 2). Table 2 gives an overview of the distribution of LACE scores among the CAP cohort. The components of the LACE index were all significantly related to all-cause 30-day readmission rates. The odds ratios of the association between the different LACE variables and the risk of 30-day all-cause readmissions (for a one-category increase within each variable) were 1.10 (95\% CI 1.04-1.16) for the length of stay, 1.11

\section{TABLE 1 Reasons for readmission after an index hospitalisation for community-acquired} pneumonia (CAP)

\begin{tabular}{lcc} 
Diagnosis & \multicolumn{2}{c}{ Number of readmissions } \\
\cline { 2 - 3 } & $\begin{array}{c}\text { Within } \mathbf{3 0} \text { days } \mathbf{n}(\% \text { of } \\
\text { readmissions) }(\mathbf{n = 6 3 6 )}\end{array}$ & $\begin{array}{c}\text { Within } \mathbf{1} \text { year } \mathbf{n}(\% \text { of } \\
\text { readmissions) (n=1851) }\end{array}$ \\
CAP & $113(17.8 \%)$ & $228(12.3 \%)$ \\
COPD & $44(6.9 \%)$ & $121(6.5 \%)$ \\
Heart failure & $42(6.6 \%)$ & $103(5.6 \%)$ \\
Chest pain & $21(3.3 \%)$ & $66(3.6 \%)$ \\
Aspiration pneumonia & $22(3.5 \%)$ & $50(2.7 \%)$ \\
Acute lower respiratory tract infection & $15(2.4 \%)$ & $43(2.3 \%)$ \\
Pleural effusion & $15(2.4 \%)$ & $19(1.0 \%)$ \\
Others & $364(57.2 \%)$ & $1221(66.0 \%)$
\end{tabular}




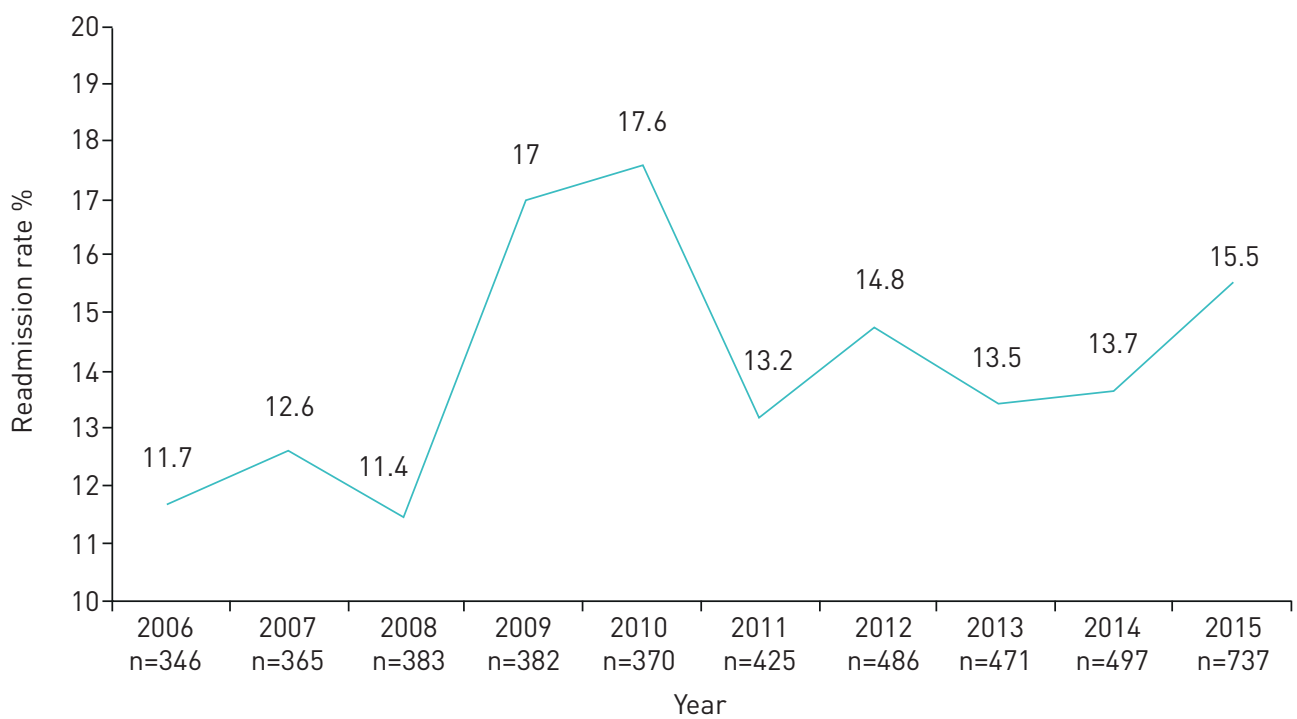

FIGURE 1 The 30-day hospital readmission rates for any diagnosis following an index hospitalisation for community-acquired pneumonia (CAP), 2006-2015. Between 2009 and 2010, Australia was affected by the global H1N1 influenza pandemic. n: number of index hospital admissions for CAP per year.

(95\% CI 1.06-1.17) for comorbidity, and 1.46 (95\% CI 1.35-1.57) for the number of emergency visits in the last 6 months. The acuity criterion from the LACE index was not relevant to our cohort as all patients were admitted through the emergency department.

The LACE index had a moderate discriminative ability to predict 30-day all-cause readmissions (C-statistic=0.6395) (figure 3). The sensitivity and specificity of LACE to predict 30-day all-cause readmissions varied based on the chosen LACE cut-off score. Table 3 gives an overview of the sensitivity and specificity for each cut-off point of the LACE index in CAP patients. Of 4508 index hospitalisations with a CAP admission, $36.4 \%$ had a LACE score $\geqslant 10$ and of 636 30-day all-cause readmissions following a hospitalisation for CAP, 348 (54.7\%) had a LACE score $\geqslant 10$.

When modifying the LACE index by replacing the acuity variable with other variables, the C-statistic for the model was 0.6345 for being male, 0.6235 for weekend admission, 0.6291 for after-hours admission, 0.6282 for summer admission, 0.6393 for winter admission, 0.6395 for COPD, 0.6393 for heart failure, 0.6395 for diabetes, 0.6395 for chronic renal failure; 0.64 for age $>60$ years, 0.6394 for age $>70$ years, and 0.6366 for age $>80$ years.

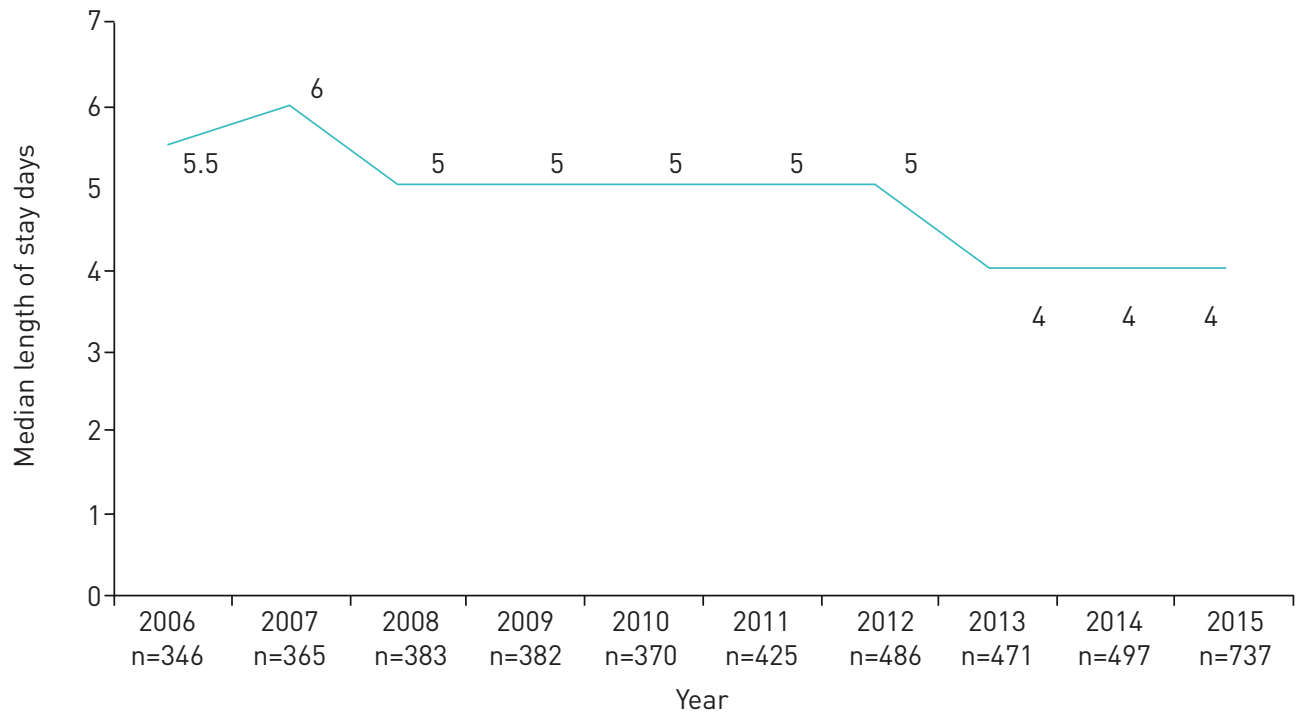

FIGURE 2 Median length of stay for index hospitalisation for community-acquired pneumonia (CAP), 2006-2015. n: number of index hospital admissions for CAP per year. 
TABLE 2 Distribution of LACE scores among patients hospitalised for community-acquired pneumonia (CAP)

LACE score

CAP admissions $n(\%)$

3

4

5

6

7

8

9

10

11

12

13

14

15

16

17

18

19
$114(2.5 \%)$

$218(4.8 \%)$

282 (6.3\%)

373 (8.3\%)

723 (16.0\%)

$671(14.9 \%)$

$486(10.8 \%)$

$437(9.7 \%)$

301 (6.7\%)

$272(6.0 \%)$

$276(6.1 \%)$

$100(2.2 \%)$

148 (3.3\%)

57 (1.3\%)

$32(0.7 \%)$

$11(0.2 \%)$

$7(0.2 \%)$

LACE: Length of stay, Acuity of admission, Comorbidities based on Charlson comorbidity score and number of Emergency visits in the last 6 months.

When only index admissions with either one of the three most common ICD-10 codes for CAP in our cohort (J18.9, J13, J18.0) were included (Supplementary Table S2), the C-statistic for all-cause readmissions substantially changed only when including index admissions with ICD-10 code J18.0 (unspecified bronchopneumonia) with a C-statistic of 0.682. However, there were only 70 index admissions with this code (1.6\% of all admissions). Including only the 3211 index hospitalisation episodes with a duration $\leqslant 7$ days ( $80.4 \%$ of all episodes) had a C-statistic of 0.6479 for all-cause readmissions.

\section{Predicting readmissions due to CAP}

The ability of the LACE index to predict 30-day readmissions due to CAP was worse than for 30-day all-cause readmissions (C-statistic $=0.5760$ versus 0.6395 ). Modifying the LACE index by replacing the acuity variable with other variables (being male; weekend admission; after-hours admission; summer or winter admission; specific comorbidities (COPD, heart failure, diabetes, and chronic renal failure); and age

FIGURE 3 Receiver operating characteristic (ROC) curve for the LACE (Length of stay, Acuity of admission, Comorbidities based on Charlson comorbidity score and number of Emergency visits in the last 6 months) index predicting 30-day readmissions after hospitalisation for communityacquired pneumonia (CAP). The ROC curve illustrates the performance of the LACE index for predicting 30-day readmission at different cut-off points. With increased sensitivity, specificity is decreased and vice versa. The area under the curve, which is equal to the $\mathrm{C}$-statistic, is 0.6353, indicating a moderate predictive ability of the model for identifying patients at risk of readmission within 30 days.

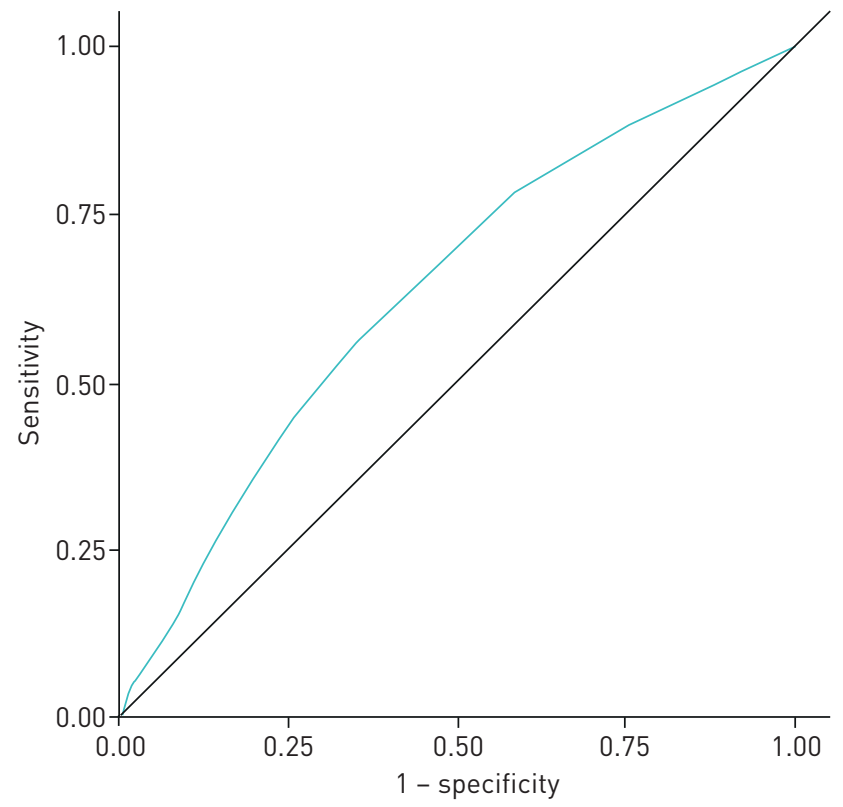


TABLE 3 Sensitivity and specificity for each cut-off point of the LACE index to predict a 30-day readmission after community-acquired pneumonia

\begin{tabular}{ccc} 
LACE score & Sensitivity & Specificity \\
\hline $\mathbf{3}$ & 1.00 & 0.00 \\
$\mathbf{4}$ & 0.99 & 0.03 \\
$\mathbf{5}$ & 0.96 & 0.08 \\
$\mathbf{6}$ & 0.93 & 0.15 \\
$\mathbf{7}$ & 0.89 & 0.24 \\
$\mathbf{8}$ & 0.79 & 0.41 \\
$\mathbf{9}$ & 0.65 & 0.56 \\
$\mathbf{1 0}$ & 0.55 & 0.67 \\
$\mathbf{1 1}$ & 0.43 & 0.76 \\
$\mathbf{1 2}$ & 0.33 & 0.82 \\
$\mathbf{1 3}$ & 0.24 & 0.88 \\
$\mathbf{1 4}$ & 0.13 & 0.93 \\
$\mathbf{1 5}$ & 0.10 & 0.95 \\
$\mathbf{1 6}$ & 0.05 & 0.98 \\
$\mathbf{1 7}$ & 0.03 & 0.99 \\
$\mathbf{1 8}$ & 0.01 & 1.00 \\
$\mathbf{1 9}$ & 0.01 & 1.00 \\
\hline Each score identifies individuals in the high-risk group for hospital readmission after 30 days, for example, \\
at 10 , admissions with a score of $\geqslant 10$ are identified as returning to hospital within 30 days by the LACE \\
index. LACE: Length of stay, Acuity of admission, Comorbidities based on Charlson comorbidity score and \\
number of emergency visits in the last 6 months.
\end{tabular}

$>60,>65,>70,>75$, and $>80$ years) respectively did not substantially change the predictive ability of the index for 30-day readmissions due to CAP.

The number of emergency visits in the last 6 months was the only tested variable that was significantly associated with an increased risk of 30-day readmissions due to CAP. This variable was associated with a C-statistic of 0.6013 and could thus better predict the risk of a 30-day readmission due to CAP than any other (combination of) the above listed variable(s) including the original LACE index.

Including only index admissions with either one of the three most common ICD-10 codes for CAP in our cohort (J18.9, J13, J18.0) did not substantially change the C-statistic for readmissions due to CAP (Supplementary Table S2). Including only the 3211 index hospitalisation episodes with a duration $\leqslant 7$ days ( $80.4 \%$ of all episodes) had a C-statistic of 0.5736 for readmissions due to CAP.

\section{Validation of ICD codes}

For a random sample of 30 patients within our cohort we reviewed the patient's medical record, including radiology and pathology reports to examine the accuracy of the diagnosis-related group (DRG) codes. We found that two patients with a DRG code for unspecified pneumonia had aspiration pneumonia based on the notes in the medical records. Overall, 10 out of the 30 patients with a pneumonia diagnosis based on clinical signs and symptoms had uncertainty regarding the finding of a pneumonic infiltrate on chest radiography.

\section{Discussion}

In this cohort, the 30-day readmission rate following a hospitalisation for CAP was 14.1\% (based on hospitalisation episodes) with $14.6 \%$ of patients having at least one 30 -day readmission. The most common reason for readmission was another episode of CAP (in 17.7\% of all 30-day readmissions), followed by COPD, heart failure and chest pain. The LACE index had only a moderate ability to predict all-cause 30-day readmissions $(\mathrm{C}$-statistic $=0.6395)$ and performed worse for 30-day readmissions due to CAP (C-statistic $=0.5760)$.

The all-cause 30-day readmission rate in patients hospitalised for CAP of $14.6 \%$ in our cohort compares to 30 -day all-cause readmission rates ranging from $11.8 \%$ to $20.8 \%$ in a systematic review of seven studies that evaluated risk prediction models for 30-day hospital readmissions in patients hospitalised for pneumonia [12]. Except for one study with a readmission rate of $11.8 \%$ that included patients with CAP only, all studies included patients with any pneumonia diagnosis. In our cohort, $2.8 \%(n=110)$ of 3996 
patients were readmitted with CAP. In a systematic review and individual patient data meta-analysis of six randomised trials that assessed adjunctive corticosteroids versus placebo in adult patients with CAP, 30-day readmissions due to CAP were $5 \%(\mathrm{n}=33$ of 748$)$ in the intervention group and $2.7 \%(18 / 758)$ in the placebo group [16].

Comparable with hospitalisations for COPD and heart failure in which the leading cause for 30-day readmissions was again COPD and heart failure respectively [9, 17], the main reason for 30-day readmissions following a hospitalisation for pneumonia was again pneumonia in our study as well as in the literature $[9,10,18]$. Whether repeat hospitalisations with pneumonia as a primary diagnosis within 30 days are caused by treatment failure, relapse or a recurrent pneumonia with a different pathogen has not been assessed in this and previous studies that evaluated readmissions in patients with pneumonia, probably also because the boundaries between the definitions of treatment failure, relapse and recurrence are often blurred and in the absence of positive microbiology results it might be impossible to determine the exact reason for another episode or a worsening episode of pneumonia.

After CAP, COPD and heart failure were the most common reasons for 30-day readmissions following an index hospitalisation for CAP in our study, a finding that is supported by the result of a systematic review of 12 studies [18]. This suggests that an episode of CAP may trigger an exacerbation of COPD [19] and increase vulnerability to subsequent cardiovascular events including myocardial infarction, congestive heart failure, arrhythmia, unstable angina and stroke [20-22], possibly as a consequence of the systemic inflammation associated with pneumonia.

Misdiagnoses of pneumonia in patients with COPD exacerbations and left heart failure may contribute to the high readmission rates noted for "pneumonia". There is evidence that the use of an early chest computed tomography scan can alter the diagnosis substantially when CAP is suspected [23, 24], suggesting that parenchymal infiltrates on chest radiography are often unspecific.

The predictive ability of models for the 30-day readmission risk following an admission for pneumonia has ranged from a C-statistic of 0.59 to 0.77 (median of 0.63) [12]. Thus, the performance of the generic LACE index in a population of pneumonia patients in our study (C-statistic of 0.6395) was slightly better than the median performance of other (more complex) risk prediction tools in pneumonia patients. The prediction model with the best discriminative ability in the literature assessed the model's performance to predict readmissions due to pneumonia (C-statistic of 0.65 ) or due to causes unrelated to pneumonia (C-statistic of 0.77 ) separately [25]. Our tested prediction models using the LACE index and other readily available variables (sex, age, comorbidities, after-hours admission, weekend admission, summer or winter admission) did not perform well for the prediction of 30-day readmissions due to CAP. The only variable significantly correlated with this risk was the number of emergency visits in the last 6 months. These findings indicate that disease specific variables during the index hospitalisation likely play a bigger role in readmissions due to CAP than in all-cause readmissions [25].

Strengths of our study were the long study duration (allowing to analyse trends in readmission rates and length of stay and how they relate over time), and the relatively large cohort of patients with CAP. A study limitation was that, as this was a single centre study, information was only available on readmissions to the same hospital where the patients had been hospitalised for their index hospitalisation. Another limitation was that the coding was often not specific for CAP, but many codes were related to not further specified pneumonia. We attempted to reduce misclassification (wrong or nonspecific ICD code for the correct diagnosis) by using additional DRG codes to exclude immunocompromised patients. The random chart review revealed that there were 10 of 30 cases with retrospectively unclear infiltrate on chest radiography, which potentially relates to the common problem (discussed above) of potential misdiagnosis of COPD exacerbation or heart failure as CAP rather than misclassification.

Risk prediction models for 30-day readmissions are of interest because they allow the identification of a "high-risk group" for targeted interventions to reduce 30-day readmissions. The evidence for interventions to reduce 30-day hospital readmissions in patients hospitalised for pneumonia is, however, sparse. A systematic review of three studies (two randomised controlled trials (RCTs) and one pseudo-RCT) on interventions to reduce hospital readmissions in patients with CAP found that a structured discharge process did not reduce hospital readmissions at 30, 90, and 180 days [26]. Medication reconciliation and follow-up telephone calls were effective strategies to reduce hospital readmissions in patients with CAP [26], whereas early mobilisation did not reduce 30-day readmissions in patients with CAP [27].

In conclusion, the ability of the LACE index to predict 30-day readmissions is at least as good or superior compared to most other pneumonia-specific risk prediction tools, but its performance is still only moderate. For the prediction of 30-day readmissions due to CAP, the performance of the LACE index and modified risk prediction models using other readily available variables is insufficient. Further research is 
required to evaluate interventions to reduce the risk of readmission in pneumonia that will also result in improved quality of life.

Conflict of interest: None declared.

Support statement: C.C. Dobler was supported by an Australian National Health and Medical Research Council fellowship (APP1123733). Funding information for this article has been deposited with the Crossref Funder Registry.

\section{References}

1 Kripalani S, Theobald CN, Anctil B, et al. Reducing hospital readmission rates: current strategies and future directions. Annu Rev Med 2014; 65: 471-485.

2 Chin DL, Bang H, Manickam RN, et al. Rethinking thirty-day hospital readmissions: shorter intervals might be better indicators of quality of care. Health Aff (Millwood) 2016; 35: 1867-1875.

3 Boulding W, Glickman SW, Manary MP, et al. Relationship between patient satisfaction with inpatient care and hospital readmission within 30 days. Am J Manag Care 2011; 17: 41-48.

4 Harb N, Foster JM, Dobler CC. Patient-perceived treatment burden of chronic obstructive pulmonary disease. Int J Chron Obstruct Pulmon Dis 2017; 12: 1641-1652.

5 Dobler CC, Harb N, Maguire CA, et al. Treatment burden should be included in clinical practice guidelines. BMJ 2018; 363: k4065.

6 Berry JG, Gay JC, Joynt Maddox K, et al. Age trends in 30-day hospital readmissions: US national retrospective analysis. BMJ 2018; 360: k497.

7 Horney C, Capp R, Boxer R, et al. Factors associated with early readmission among patients discharged to post-acute care facilities. J Am Geriatr Soc 2017; 65: 1199-1205.

8 Dobler CC. Defragmenting health care for patients with chronic obstructive pulmonary disease. AMJ 2016; 9 ; $185-188$.

9 Jencks SF, Williams MV, Coleman EA. Rehospitalizations among patients in the Medicare fee-for-service program. N Engl J Med 2009; 360: 1418-1428.

10 Dharmarajan K, Hsieh AF, Lin Z, et al. Diagnoses and timing of 30-day readmissions after hospitalization for heart failure, acute myocardial infarction, or pneumonia. JAMA 2013; 309: 355-363.

11 Kansagara D, Englander H, Salanitro A, et al. Risk prediction models for hospital readmission: a systematic review. JAMA 2011; 306: 1688-1698.

12 Weinreich M, Nguyen OK, Wang D, et al. Predicting the risk of readmission in pneumonia. a systematic review of model performance. Ann Am Thorac Soc 2016; 13: 1607-1614.

13 van Walraven C, Dhalla IA, Bell C, et al. Derivation and validation of an index to predict early death or unplanned readmission after discharge from hospital to the community. CMAJ 2010; 182: 551-557.

14 Quan H, Li B, Couris CM, et al. Updating and validating the Charlson comorbidity index and score for risk adjustment in hospital discharge abstracts using data from 6 countries. Am J Epidemiol 2011; 173: 676-682.

15 Dobler CC, Waterer G. Healthcare-associated pneumonia: a US disease or relevant to the Asia Pacific, too? Respirology 2013; 18: 923-932.

16 Briel M, Spoorenberg SMC, Snijders D, et al. Corticosteroids in patients hospitalized with community-acquired pneumonia: systematic review and individual patient data meta-analysis. Clin Infect Dis 2018; 66: 346-354.

17 Hakim MA, Garden FL, Jennings MD, et al. Performance of the LACE index to predict 30-day hospital readmissions in patients with chronic obstructive pulmonary disease. Clin Epidemiol 2018; 10: 51-59.

18 Prescott HC, Sjoding MW, Iwashyna TJ. Diagnoses of early and late readmissions after hospitalization for pneumonia. A systematic review. Ann Am Thorac Soc 2014; 11: 1091-1100.

19 Roca M, Verduri A, Corbetta L, et al. Mechanisms of acute exacerbation of respiratory symptoms in chronic obstructive pulmonary disease. Eur J Clin Invest 2013; 43: 510-521.

20 Eurich DT, Marrie TJ, Minhas-Sandhu JK, et al. Risk of heart failure after community acquired pneumonia: prospective controlled study with 10 years of follow-up. BMJ 2017; 356: j413.

21 Perry TW, Pugh MJ, Waterer GW, et al. Incidence of cardiovascular events after hospital admission for pneumonia. Am J Med 2011; 124: 244-251.

22 Wang Y, Eldridge N, Metersky ML, et al. National trends in patient safety for four common conditions, 2005-2011. N Engl J Med 2014; 370: 341-351.

23 Claessens YE, Debray MP, Tubach F, et al. Early chest computed tomography scan to assist diagnosis and guide treatment decision for suspected community-acquired pneumonia. Am J Respir Crit Care Med 2015; 192: 974-982.

24 Prendki V, Scheffler M, Huttner B, et al. Low-dose computed tomography for the diagnosis of pneumonia in elderly patients: a prospective, interventional cohort study. Eur Respir J 2018; 51: 1702375.

25 Capelastegui A, Espana Yandiola PP, Quintana JM, et al. Predictors of short-term rehospitalization following discharge of patients hospitalized with community-acquired pneumonia. Chest 2009; 136: 1079-1085.

26 Domingo GR, Reyes FC, Thompson FV, et al. Effectiveness of structured discharge process in reducing hospital readmission of adult patients with community acquired pneumonia: a systematic review. JBI Libr Syst Rev 2012; 10: $1086-1121$.

27 Melgaard D, Baandrup U, Bogsted M, et al. Early mobilisation of patients with community-acquired pneumonia reduce length of hospitalisation: a pilot study. J Phys Ther Sci 2018; 30: 926-932. 